\title{
Integrating metabolic and evolutionary engineering for enhanced propionic acid production under cross stress: a multi-omics perspective
}

Tingting Liu

Nanjing Tech University

Qianru Zhao

Nanjing Tech University

Yang Li

Nanjing Tech University

Liying Zhu

Nanjing Tech University

Ling Jiang ( $\nabla_{\text {jiangling@njtech.edu.cn ) }}$

Nanjing Tech University https://orcid.org/0000-0001-6625-5557

He Huang

Nanjing Tech University

Research

Keywords: Propionibacterium acidipropionici, Propionic acid, Metabolic and evolutionary engineering, Cross stress, Omics analysis

Posted Date: December 16th, 2019

DOI: https://doi.org/10.21203/rs.2.18982/v1

License: (c) (i) This work is licensed under a Creative Commons Attribution 4.0 International License. Read Full License 


\section{Abstract}

Background: Propionic acid (PA), a potential building block for C3-based bulk chemicals, is used as a food preservative and antifungal agent because of the antimicrobial properties of its calcium-, potassium-, and sodium salts, as well as in the manufacture of pharmaceuticals, perfumes, pesticides and fungicides. However, industrial development of PA is seriously inhibited by oxygen stress, acid stress and glucose-induced osmotic stress concentration on account of the characteristic of Propionibacterium acidipropionici. To alleviate inhibition and increase PA production, enhancement $P$. acidipropionici tolerance to cross stress may be an effective strategy.

Results: In this study, we first performed a combination of metabolic engineering (deletion of $/ d h$ and poxB and overexpression of $\mathrm{mmc}$ ) with evolutionary engineering (selection under oxygen stress, acid stress and osmotic stress) in P. acidipropionici. The results indicated the mutants received superior physiological activity, especially the mutant III exhibited steady 1.5-3.5 folds higher growth property and further $37.1 \%$ PA titer and $37.8 \%$ PA productivity increase than the wildtype. Moreover, omics analysis revealed the determinants such as Dps, GroES, dnaK, ADI and GAD referred to the acid adaptation of microbes were positively mobilized. ABC-type glycine betaine referred to the adaptability to osmotic stress was detected to be 2-4 folds up-regulated. More than 2-fold down-regulation of NADH oxidase and almost 3-fold up-regulation of SOD and POD were observed in three mutants. Moreover, an approximately 2.5-fold upregulation of $\mathrm{mmc}$ was also found.

Conclusion: The multi-omics analysis revealed the multidirectional variation tendency of $P$. acidipropionici under cross stress and provided in-depth insights into the mechanism of tolerance and high production of PA, which layed the foundation for construction of microbial cell factories.

\section{Background}

Currently, the traditional fossil-fuel-based PA synthesis is becoming increasingly less desirable due to energy shortages, environmental pollution and the desire for sustainable development, while the fermentation of PA from renewable resources using Propionibacterium has unique advantages [1]. As the major production strains, the members of the genus Propionibacterium are generally recognized as safe [1]. P. acidipropionici, P. shermanii and P. freudenreichii are capable of utilizing a wide range of carbon sources to produce PA, including soy molasses [2], corn stover hydrolysate [3], sorbitol [4], glycerol and glucose [5] and whey lactose [6]. To date, many strategies have been developed to improve the yield of PA, including high density fermentation [7], immobilization of Propionibacterium cells [8], iNTRODUCTION OF BIOCOMPATIBLE SMALL MOLECULE [6], reduction of by-product [9], controlled pH-shift in fed-batch culture [10] and engineering of metabolic pathways [11]. However, in addition to the common problems of industrial fermentation such as end-product inhibition and by-product accumulation, the anaerobic growth of Propionibacterium spp. means that the culture conditions for PA production must be strictly controlled [12]. Although the creation of an anaerobic environment is no longer as challenging due to the development of production technology, the cost of maintaining anaerobic conditions is high and 
incompatible with the concept of developing a green economy. PA is synthesized by Propionibacterium spp. via the dicarboxylic acid pathway, which also generates byproducts such as lactic acid (LA) and acetic acid (AA) [9]. Thus, the accumulation of acid products during fermentation easily results in an acidic environment, affecting cell activity and inhibiting metabolism, which precludes industrial-scale PA production. Moreover, hypertonic solutions casused by HIGHER SUBSTRATE CONCENTRATION tend to dehydrate cells and inhibit their growth and reproduction $[13,14]$. Consequently, enhancing the tolerance of Propionibacterium spp. to oxygen, acid and hypertonic solutions is considered as an effective strategy to alleviate the inhibition and has triggered a research hotspot.

The adaptation of bacteria to a stressful environment can lead to the accumulation of mutations that confer a fitness advantage when exposed to a different stressor, which was named 'cross-stress' protection $[15,16]$. Therefore, the application of cross stress in adaptive evolution, especially in combination with metabolic engineering, has certain advantages for industrial strain development. Metabolic engineering has been used as an effective strategy for increasing PA production. Liu et al. improved the PA titer of P. jensenii by $34.7 \%$ by overexpressing phosphoenolpyruvate carboxylase (ppc) and deleting lactate dehydrogenase (Idh) [11]. Guan et al. obtained a $12.2 \%$ increase of PA by constructing a P. acidipropionici strain with simultaneous deletions of Idh1, Idh2 and pyruvate oxidase (poxB) [17]. However, combining metabolic engineering with laboratory evolution to improve the synthesis of PA has not been published, and may be a successful strategy. Furthermore, omics technologies are able to identify crucial genes and metabolites, providing valuable information that reflects stress-induced changes and the intricate interplay between organisms and the environment $[18,19]$. For example, comparative genomic and transcriptomic analyses of $P$. acidipropionici revealed the molecular mechanisms of acid tolerance, while proteomics analyses revealed how P. acidipropionici respond to propionic acid stress [20].

Therefore, in this study, P. acidipropionici CGMCC 1.2232 was engineered by combining metabolic engineering with evolutionary engineering to adjust the direction of metabolic flow to maximize the production of PA and improve the adaptability to environmental stresses. To probe the variation footprints of determinants involved in the tolerance mechanisms under cross stress and mechanism of high PA production, multi-omics analysis of wild-type P. acidipropionici and the improved mutants was performed and then integrated, providing valuable guidance for the industrial production of PA.

\section{Results And Discussion}

\section{Metabolic and evolutionary engineering, and the growth rates of mutant strains}

The Idh and poxB genes, which are main contributors of the by-products acetate and lactate [21], were deleted. Additionally, the methylmalonyl-CoA carboxyltransferase $(\mathrm{mmc})$ gene from P. acidipropionici, which controls the carbon flow into the propionate-producing Wood-Werkman cycle [22] was overexpressed. These manipulations yielded the strains P. acidipropionici- $\Delta$ ldh, P. acidipropionici- $\Delta$ ldh- 
$\Delta \mathrm{poxB}$, and P. acidipropionici- $\Delta \mathrm{ldh}-\Delta \mathrm{poxB}+\mathrm{mmc}$, respectively. In evolutionary engineering, specific stress is applied gradually on the basis of the growth rate of the adapted strain, which gives a buffer period so that the strain can adequately mobilize the in vivo response mechanisms to resist stress (Fig. 1). During the evolution of P. acidipropionici- $\Delta \mathrm{ldh}$ into the mutant I, the growth of the experimental strain displayed a peak at $\mathrm{OD}_{600} 17.1$ on the fourth day of culture, followed by a downward trend because of the decline of $\mathrm{pH}$ and the increase of glucose concentration. Subsequently, the growth rate of P. acidipropionici- $\Delta \mathrm{ldh}$ tended to be stable at $\mathrm{OD}_{600} 13.9$ and began to descend when the $\mathrm{pH}$ was decreasing again (6 to 5), and was ultimately stable at $\mathrm{OD}_{600} 15.9$. From $\mathrm{P}$. acidipropionici- $\Delta \mathrm{ldh}-\Delta$ poxB to the mutant II (deletion of poxB followed by evolutionary engineering), the formation of the subsequent two peaks at $\mathrm{OD}_{600} 16.5$ and 15.4 on the 6th and 15th day corresponded to the progressive deepening of the levels of single stress factors. From P. acidipropionici- $\Delta \mathrm{ldh}-\Delta \mathrm{poxB}+\mathrm{mmc}$ to the mutant III (overexpression of mmc followed by evolutionary engineering), the growth rate of the strains started to pick up a steady upward trend, and the strain showed a normal growth curve after supplementation with fresh medium. The highest $\mathrm{OD}_{600}$ was 14.6 , while the lowest $\mathrm{OD}_{600}$ of 9.8 was observed with $\mathrm{pH} 4,120 \mathrm{~g} \cdot \mathrm{L}^{-1}$ glucose and $21 \%$ oxygen sparging. These results indicate that the experimental strain could still maintain a considerable growth rate under the superimposed cross stress, even when exposed to simultaneously increasing stress levels. This indicated that the strain had developed a certain level of 'cross-stress' protection and acquired a fitness advantage based on the gradual application of stress by artificial control so that it could deal with cross stress.

To further investigate the growth rate of the strain obtained by metabolic and evolutionary engineering, the wild type and mutant III were compared. Compared with the $0.09 \mathrm{~h}^{-1}$ specific growth rate of the wild type under normal fermentation conditions ( $\mathrm{pH} 7$ ), mutant III had a significant improvement at $0.14 \mathrm{~h}^{-1}$, which was apparently higher than the $0.131 \pm 0.007 \mathrm{~h}^{-1}$ previously reported for growth on soy molasses and soy molasses hydrolysate [2]. However, when the strains were exposed to low pH, the growth of both the wild type and the mutant III was suppressed accordingly. Nevertheless, the growth of the mutant III was significantly superior to that of the wild type. With the decrease of $\mathrm{pH}$, the growth rate difference between the wild type and mutant III gradually increased, and mutant III could maintain normal growth, while the growth of the wild type was clearly suppressed (Fig. S1A). As the concentration of glucose in the medium increased, the difference of the growth rate between the wild type and mutant III gradually increased. At normal glucose concentrations ( $30 \mathrm{~g} \cdot \mathrm{L}^{-1}$ glucose), the specific growth rate of the wild type was about $0.09 \mathrm{~h}^{-1}$, while that of mutant III reached $0.2 \mathrm{~h}^{-1}$, which was 1.4 -fold higher than the previously reported value for the same glucose concentrations [23]. While the wild type almost stopped growing at a high glucose concentration of $120 \mathrm{~g} \cdot \mathrm{L}^{-1}$, mutant III could maintain growth at $\mathrm{OD}_{600} 0.4$ (Fig. S1B). Futhermore, the strain exhibited similar growth characteristics when exposed to oxygen stress compared with acid stress and osmotic stress. A 2-fold growth rate difference has observed under anaerobic fermentation conditions (oxygen content $0.001 \%$ ). Under these conditions, the specific growth rate of mutant III reached $0.18 \mathrm{~h}^{-1}$, while that of the wild type was $0.09 \mathrm{~h}^{-1}$. By contrast, with $21 \%$ oxygen content in the sparged gas mixture, the specific growth rate of the wild type was only $0.03 \mathrm{~h}^{-1}$, while that 
of mutant III was $0.05 \mathrm{~h}^{-1}$ (Fig. S1C). As a result, the growth performance of mutant III under cross stress was clearly superior to that of the wild type. Importantly, the generation of the mutant III has proved that evolutionary engineering is an effective method for improving the resistance of $\mathrm{P}$. acidipropionici to cross stress.

\section{PA fermentation using the engineered P. acidipropionici}

Batch fermentations were carried out using wild-type P. acidipropionici as well as the mutants I, II and III to investigate their PA production capacity. As shown in Table 1, under the optimal culture conditions $(\mathrm{pH}$ $7,99 \% \mathrm{~N}_{2}, 5 \mathrm{~g} \cdot \mathrm{L}^{-1}$ glucose), the PA titer and productivity of mutant I were increased by 12.4 and $13.5 \%$ compared to the wild type. Its performance was therefore also significantly better than that of P. jensenii$\Delta$ ldh with 1.6 and $8.1 \%$ enhancement [11], respectively. At the same time, a $68.8 \%$ of decrease of LA yield $\left(3.06 \pm 0.82\right.$ vs. $\left.1.04 \pm 0.17 \mathrm{~g} \cdot \mathrm{L}^{-1}\right)$ was obtained. However, there was a $3 \%$ increase of AA titer $(5.24 \pm 0.83$ vs. $5.63 \pm 0.62 \mathrm{~g} \cdot \mathrm{L}^{-1}$ ), which may be related to the deletion of $\mathrm{Idh}$, which increased carbon flow from pyruvate into the synthesis of LA [21]. The PA production of the mutant II only increased by $3.2 \%$, while the titers of $A A$ and $L A$ were both decreased by more than $70 \%$, which was consistent with the variation tendency of the PA, LA and AA titers of P. acidipropionici- $\triangle$ poxB- $\Delta$ ldh [17]. A further $37.1 \%$ increase of PA titer $\left(28.1 \pm 0.96 \mathrm{~g} \cdot \mathrm{L}^{-1} \mathrm{vs} .38 .7 \pm 1.14 \mathrm{~g} \cdot \mathrm{L}^{-1}\right)$ and $37.8 \%$ increase of PA productivity $\left(0.216 \pm 0.006 \mathrm{~g} \cdot \mathrm{L}^{-1}\right.$ vs. $0.298 \pm 0.008 \mathrm{~g} \cdot \mathrm{L}^{-1} \cdot \mathrm{h}^{-1}$ ) was achieved in the mutant III. This increase was much more significant than the reported value achieved via the overexpression of $\mathrm{mmc}$ in $\mathrm{P}$. freudenreichii in batch fermentation [23]. At the same time, there was a 78.6 and $87.8 \%$ decrease of the byproduct yields because of the deletion of Idh and poxB, and the overexpression of mmc drove the carbon flow into the synthesis of PA to the greatest extent.

Table 1

Analysis of microbial production using engineered P. acidipropionici in batch fermentation.

\begin{tabular}{|lllll|}
\hline Strain & $\begin{array}{l}\text { PA titer } \\
\left(\mathbf{g} \cdot \mathbf{L}^{-1}\right)\end{array}$ & $\begin{array}{l}\text { LA titer } \\
\left(\mathbf{g} \cdot \mathbf{L}^{-1}\right)\end{array}$ & $\begin{array}{l}\text { AA titer } \\
\left(\mathbf{g} \cdot \mathbf{L}^{-1}\right)\end{array}$ & $\begin{array}{l}\text { PA productivity } \\
\left(\mathbf{g} \cdot \mathbf{L}^{-1} \cdot \mathbf{h}^{-1}\right)\end{array}$ \\
\hline WT & $28.1 \pm 0.96$ & $3.06 \pm 0.82$ & $5.24 \pm 0.83$ & $0.216 \pm 0.006$ \\
\hline $\begin{array}{l}\text { Mutant } \\
\square\end{array}$ & $31.8 \pm 0.85$ & $1.04 \pm 0.17$ & $5.63 \pm 0.62$ & $0.245 \pm 0.007$ \\
\hline $\begin{array}{l}\text { Mutant } \\
\square\end{array}$ & $29.4 \pm 0.59$ & $0.89 \pm 0.06$ & $0.78 \pm 0.04$ & $0.226 \pm 0.006$ \\
\hline $\begin{array}{l}\text { Mutant } \\
\square\end{array}$ & $38.7 \pm 1.14$ & $0.78 \pm 0.05$ & $0.69 \pm 0.05$ & $0.298 \pm 0.008$ \\
\hline
\end{tabular}




\section{Comparative omics analysis of the wild type and the mutants I, II, III}

The adaptation of microbes to environmental stress can produce beneficial phenotypes through random genomic mutations and subsequent positive selection [24]. In this study, the resistance mechanism of the strains to cross stress may be elucidated by analyzing the transcriptome, proteome and metabolome of the different $\mathrm{P}$. acidipropionici strains. The original reads of transcriptome and proteome have been deposited in the NCBI Sequence Read Archive (Accession No. SRR10597965) and PRIDE database (Accession No. PXD016616), respectively. The results showed that the three evolved mutants reflected the dynamic evolution of the resistance mechanism of P. acidipropionici based on the wild type. At the transcriptional level, the differentially expressed genes (DEGs) predicted in the three groups compared to the wild type are shown in Fig. 2a. A total of 166 DEGs were identified in the mutant I, with 72 up- and 94 down-regulated genes. Similarly, 165 DEGs were identified in mutant II (85 up- and 80 downregulated genes) and 102 in mutant III (29 up- and 73 downregulated genes). The MA plot and volcano plot (Fig. 2b) show the distribution of the DEGs between the two groups of samples. As can be seen in the Venn diagram (Fig. 2c), there were 50 identical genes that were differentially expressed, meaning that a considerable number of shared or similar metabolic pathways were shared between the three mutants and the wild type. A total of 2,158 proteins were identified in the proteomic analysis (Fig. 2d). There were 343,260 , and 226 differentially expressed proteins (DEPs) in the mutants I, II, and III, respectively. Among these, 217,176 , and 147 proteins were up- while 126, 84, and 79 were downregulated, respectively. Likewise, we discovered that 72 proteins involved in the same function were differentially expressed in the three mutants. The Principal component analysis (PCA) score plot was performed to show the intracellular metabolism differences of the wild type and the mutant III (three parallel samples in each group) [25]. As shown in Fig. 3a, the strains obtained were clearly divided into four groups and the data sample point dispersion of four groups was relatively great, which indicating the metabolic profile of four groups was significantly different. Figure $3 \mathrm{~b}$ revealed 34 significantly differentially abundant intracellular metabolites, which were mainly involved in the three central metabolic pathways, including glycolysis (Dglucose, trehalose), the tricarboxylic acid cycle (malic acid), the pentose phosphate pathway (phosphoric acid), and amino acid metabolism (glycine, threonine and serine) [26]. There were a total of 17 up- and 10 downregulated metabolites in mutant I, 21 up- and 13 downregulated metabolites in the mutant II, as well as 21 up- and 12 downregulated metabolites in mutant III. Combined with the sample correlation analysis (Fig. 4), the data revealed that mutant I had the lowest similarity with the wild type, while mutant III had the highest. From the perspective of difference, mutant I showed the most dramatic changes and the most intense responses to cross stress, while mutant III was the opposite.

Based on the transcriptome sequencing results, the $\mathrm{GO}$ enrichment analysis of the DEGs was conducted as shown in Fig. 5. DEGs involved in the categories metabolic process, single-organism process in biological process, membrane and membrane part in cellular component, catalytic activity, binding and transporter activity in molecular function were all enriched with false discovery rate (FDR) q-values of less than 0.05 in the three mutants compared with the wild type. Among them, the membrane part and 
catalytic activity were significantly different. This suggested that the DEGs involved in these items were positively responsive, especially the major annotated categories membrane part and catalytic activity, which were most significantly affected by the cross stress. Moreover, it is worth noting that the DEGs were only downregulated related to transporter activity in the mutant III. The adverse cross stress conditions impacted 97 KEGG pathways in the mutant II, 84 KEGG pathways in the mutant I, and only 44 KEGG pathways in the mutant III. The top 20 enriched KEGG pathways (corrected P-value $<0.05$ ) were displayed in the scatter plot (Fig. 6). Compared with the wild type, genes related to carbon fixation pathways involving energy metabolism were upregulated in the mutants I and II, while they disappeared from the data of mutant III. Interestingly, only five metabolic pathways with upregulated genes were enriched in the mutant III, indicating that this mutant has become a mature, highly tolerant mutant on the basis of the mutants I and II. Therefore, in order to adapt to the high glucose, low pH and the aerobic fermentation environment, the mutants increased their tolerance ability to environmental stress by changing the gene expression of the relevant metabolic pathways to maintain growth and metabolism under environmental stress.

The distribution of DEPs quantified in the GO secondary annotation was statistically analyzed to gain insights into the potential functional implications for stress tolerance (Fig. 7). The categories metabolic process $(46.88 \%, 51.72 \%, 38.81 \%)$ in biological process, membrane $(31.17 \%, 27.27 \%, 35.48 \%)$ in cellular component, as well as catalytic activity (56.99\%, 61.90\%, 58.52\%), and binding (33.16\%, 29.93\%, 27.41\%) in molecular function were enriched in the mutants I, II and III, respectively, which was roughly consistent with the GO enrichment analysis of the DEGs. However, the mutant I no longer showed the most obvious changes as was the case at the transcriptome level. This result suggested that major functional categories of the strain involved in regulation remained largely unchanged under adverse environmental conditions, both at the gene and protein levels. To further determine the DEPs more likely to be mobilized to resist stress, we enriched the DEPs. In the GO enrichment (Fig. 8A), we observed that upregulated DEPs were involved in glutathione transferase activity and FAD binding in the mutant I, glycerol metabolic process and alditol metabolic process in the mutant II, while they were involved in cysteine biosynthetic process and sulfate reduction in the mutant III. In the KEGG enrichment (Fig. 8B), the microbial metabolism in diverse environment-related pathways were enriched (1.64-fold, 1.79-fold, 1.66-fold) in the mutants I, II and III, respectively. Degradation of aromatic compounds and bacterial chemotaxis were detected to be significantly enriched in the mutants I and II, while sulfur metabolism was found in the mutant III, demonstrating that this mutants developed positive responses to the adverse environment. As can be seen in Fig. 9, at the metabolic level, carboxylic acids metabolism such as butanoate and pyruvate, methane metabolism, amino acid metabolism such as glycine, serine and threonine, as well as cysteine and methionine in the three mutants were all significantly affected when subjected to cross stress. The pathway analysis results implied the mutants might modulate the stress response mechanisms against to abiotic stress. 


\section{Differentiation analysis of the determinants related to cross stress of the wild type and the mutants I, II and III}

In order to understand the detailed resistance mechanism of P. acidipropionici subjected to cross stress, we investigated the variation footprints of the changes from the wild type to mutant III, from the DNA to metabolite levels. Maintaining pH homeostasis is the main challenge under acid stress, which may be supported by ATP metabolism and active transport of protons. The $\Delta \mathrm{pH}$ in the cell can drive the generation of proton motive force (PMF) to enhance cellular protonation [27]. Previous studies have shown that specific genes are commonly expressed by Gram-positive neutrophilic bacteria during acid stress [28]. In this study, genes related to the ATPase-coupled transmembrane transporter activity were confirmed to be downregulated to retard protonation based on the transcriptome analysis in the three mutants. The interconversion between NADH and NAD is associated with ATP metabolism in the coupling of proton levels. The NADH levels were reduced 2.97-, 2.80-, and 3.54-fold, while those of NAD were reduced 8.11-, 6.50-, and 5.81-fold in three mutants, respectively. Moreover, the determinants of the regulation of $\mathrm{pH}$ homeostasis, such as cations and inorganic ions, were also discovered in the three mutants. $A B C$ transporters were transcriptionally upregulated in the three mutants, which may mediate the translocation of large amounts of substrate across the cell membranes [29]. The Dps protein, which protects cells against acid damage [30], was expressed 3.47-fold more at the transcriptional level in the mutant I, as well as 2.15-, 1.89-, and 3.49-fold more at the proteome level in three mutants compared to the wild type, respectively. The genes GroES and dnaK related to the acid adaptation of bacteria were also differentially expressed at the protein level [31]. GroES were upregulated in all three mutants. DnaK was downregulated in mutant I, but upregulated in the mutants II and III. Furthermore, GroES was found downregulated in the mutants I, II according to transcriptional analysis. Amino acid metabolism plays an important role in the acid tolerance of bacteria [32]. The arginine deiminase (ADI) system, which consists of arginine deiminase, carbamate kinase (CK) and ornithine transcarbamoylase, has been proved to exist in the genus Propionibacterium [33,34]. This system is activated under acidic conditions because of its contribution to $\mathrm{pH}$ homoeostasis [28]. In this study, the expression of ADI and CK were not detected in RNA-seq and metabolome analysis, but the proteins were detected in the proteome. Moreover, the glutamate decarboxylase (GAD) pathway, which can consume $\mathrm{H}^{+}$[35], did not show significant differential expression in the mutant II II, and III, respectively. Therefore, the trajectory of change of ADI and GAD remained consistent, whereby mutant II showed more positive regulation of ADI and GAD, while mutant I was more positive in the regulation of CK.

We observed that the ABC-type glycine betaine transport system, which is related to the adaptability to osmotic stress [36], was not observed transcriptionally regulated in the three mutant, but upregulated 2.81-, 2.06-, 4.51-fold on the proteomic level in the mutant I, II, and III. Additionally, it was reported that high osmolarity can induce the oxidative stress regulons [37, 38]. It has been reported that $\mathrm{H}_{2} \mathrm{O}_{2}$ can be produced by NADH oxidase in the cell membrane when anaerobes are exposed to oxygen, posing a threat to cell survival [39]. Interestingly, we only observed a significant upregulation of NADH oxidase by proteome analysis with 2.00-, 2.05-, 2.05-fold in three mutants. In addition, the expression of superoxide 
dismutase (SOD) and peroxidase (POD) was upregulated in the protein level. Notably, there was a 2.99fold upregulation of SOD expression in the mutant III and 3.18-fold upregulation of POD expression in the mutant II. These results indicate that the mutants also adapted to oxidative stress. In addition, as a bioprotective agent, trehalose exhibits prominent biological functions against adverse abiotic stress, such as oxygen radicals and acid stress [6]. Found by metabolomics, the level of trehalose was a persistent upward trend from the wild type to the mutant III, especially the mutant III with the most significant level in the content compared to the wild type. It verified that the mutant III was subjected to the relatively large degree of cross stress. Some metabolites such as serine, aspartic acid, glycine and trimethylol propane were only observed in the mutant II and III, while methanediimine, glycol acid and ethylaminewhich were only observed in the wild type.

Pyruvate from glucose or glycerol is a vital precursor in the metabolic pathway of propionic acid synthesis in Propionibacterium spp. However, the pyruvate is also converted into byproducts, most importantly into acetate by poxB and lactate by Idh [18]. Therefore, poxB and Idh were selected to be deleted to reduce the metabolic flux into the formation of acetate and lactate. However, another source of acetate synthesis is the conversion of pyruvate to acetyl-CoA. This indicated that the deletion of poxB did not completely block the carbon flux into acetate synthesis, which was also confirmed by the fermentation data. Unsurprisingly, no differences of Idh and poxB were found in the omics results. The lactate permease involved in lactate transport driven by a proton motive force was respectively upregulated 3.6-, 2.6- and 1.88-fold in the mutants according to the proteomic analysis. Therefore, decreased lactate synthesis promoted lactate permease activity to maintain the intracellular metabolic homeostasis [40]. For PA synthesis, pyruvate is converted to oxaloacetate, which finally flows to PA via $\mathrm{mmc}$ through the transfer of one carbon from methylmalonyl-CoA to pyruvate [41]. The overexpression of mmc led to 1.12- and 2.73-fold, as well as 1.34- and 2.58-fold upregulation in the mutant II and III according to the RNA-Seq and proteomic analysis, respectively. However, there were no differences in mutant I, indicating that the increase of carbon flux to PA formation due to the deletion of Idh could be neglected. This was corroborated by the fermentation results. Moreover, succinate dehydrogenase converts fumarate to succinate, which is also an important precursor of PA. It was found to be 2.88- and 2.63- upregulated at the transcriptional level in the mutant I and II, as well as 2.94-, 2.29- and 1.85-fold at the protein level in the three mutants, respectively. Interestingly, pyruvate carboxylase, which converts pyruvate to oxaloacetate, likewise was only found to be upregulated in mutant III, 1.66 -fold at the protein level, which explains why the omics results for $\mathrm{mmc}$ and succinate dehydrogenase were inconsistent. Moreover, the variation trend of the contents of PA, LA and AA at the metabolic level from the wild type to the mutant III was consistent with the fermentation results.

\section{Conclusions}

Rational P. acidipropionici mutants were constructed by metabolic engineering to promote carbon flow to the PA production. Subsequently, adaptive laboratory evolution of the mutants was conducted by the application of cross stress. The mutant III had a 1.5 to 3.5 -fold higher growth rate, as well as a $37.1 \%$ increase of PA titer and increase of $37.8 \%$ PA productivity compared to the wild type. Omics analysis 
revealed that the determinants related to the tolerance mechanism and PA production were actively mobilized through increased expression. These results offer a better understanding of the mechanism of increased PA production, which lays a foundation for the construction of advanced microbial cell factories for the industrial fermentation of PA.

\section{Materials And Methods}

\section{Microorganisms and culture media}

All strains, plasmids and primers used in this study are listed in Table S1. P. acidipropionici CGMCC1.2232 was propagated anaerobically at $30^{\circ} \mathrm{C}$ in culture medium containing the following: yeast extract $5 \mathrm{~g} \cdot \mathrm{L}^{-1}$; peptone $5 \mathrm{~g} \cdot \mathrm{L}^{-1}$; dipotassium hydrogen phosphate $0.25 \mathrm{~g} \cdot \mathrm{L}^{-1}$; manganese sulfate 0.05 $\mathrm{g} \cdot \mathrm{L}^{-1}$; glucose $5 \mathrm{~g} \cdot \mathrm{L}^{-1}$. Resazurin was added to a final concentration of $0.05 \%$ as an oxygen indicator. Escherichia coli DH5a was used for cloning and plasmid propagation, and was grown aerobically in Luria-Bertani (LB) medium at $37^{\circ} \mathrm{C}$. E. coli S17-1 ( $\lambda$ pir) was used as the donor strain to transform P. acidipropionici via conjugation. All media were sterilized through autoclaving ( $121^{\circ} \mathrm{C}$ for $20 \mathrm{~min}$ ), after which $50 \mu \mathrm{g} \cdot \mathrm{mL}^{-1}$ ampicillin, kanamycin, and erythromycin or $25 \mu \mathrm{g} \cdot \mathrm{mL}^{-1}$ spectinomycin was added when necessary.

Genetic manipulation and evolutionary engineering

The chromosomal target genes were deleted seamlessly according to the method we reported previously [6]. The up- and down-stream regions flanking the lactate Idh and pyruvate oxidase poxB genes were amplified using the genome of $\mathrm{P}$. acidipropionici as the template. The resulting products with the $2.0 \mathrm{~kb}$ $\mathrm{Smr} / \mathrm{Spcr} \Omega$ cassette were amplified using the high copy vector pBluescriptll SK+ (Stratagene). The Kan ${ }^{\mathrm{R}}$ suicide vector pJP5603 was selected to construct the recombinant plasmids pJLDH and pJPOXB that were transferred into P. acidipropionici cells by conjugation using the donor strain E. coli S17-1 ( $\lambda$ pir). The overexpression of genes was conducted as described before [6]. The methylmalonyl-CoA carboxyltransferase $(\mathrm{mmc})$ gene was amplified from the genome of P. acidipropionici using the primers mmc-for 1 and mmc-rev1. The shuttle vector pBRESP36A was selected to construct the mmc overexpression plasmid pBMMC that was transferred into P. acidipropionici via conjugation. Evolutionary engineering of $P$. acidipropionici mutants was carried out using cross stress in the form of different $\mathrm{pH}$ values $(7,6,5,4)$, glucose concentrations $\left(60 \mathrm{~g} \cdot \mathrm{L}^{-1}, 90 \mathrm{~g} \cdot \mathrm{L}^{-1}, 120 \mathrm{~g} \cdot \mathrm{L}^{-1}\right)$ and oxygen flux values $\left(99.999 \% \mathrm{~N}_{2}, 79 \% \mathrm{~N}_{2}+11 \% \mathrm{CO}_{2}+10 \% \mathrm{O}_{2}, 79 \% \mathrm{~N}_{2}+21 \% \mathrm{O}_{2}\right)$ during anaerobic cultivation in a $2 \mathrm{~L}$ NBS fermenter at $30^{\circ} \mathrm{C}$ and $120 \mathrm{rpm}[42,43]$. Samples were taken every $24 \mathrm{~h}$ to assess cell growth and stress tolerance. Fresh medium was added to maintain a normal $2 \mathrm{~L}$ continuous culture system. Cross stress was applied stepwise to maintain the cells at an $\mathrm{OD}_{600}$ above 1.0. The final mutant was then used as the experimental group, and the wild-type was considered as the control group. The two groups were cultured under single stress $(\mathrm{pH}$, glucose concentration, oxygen flux) to evaluate cell growth and compare their stress tolerance.

Batch fermentation of PA using the engineered P. acidipropionici 
For propionic acid fermentation, strains were pre-cultured in $100 \mathrm{~mL}$ anaerobic bottles with $50 \mathrm{~mL}$ medium with different $\mathrm{pH}$, glucose concentrations and oxygen flux until the $\mathrm{OD}_{600}$ reached 2.0. Then, $5 \%$ of seed liquid was used to inoculate a 2-L NBS fermenter with corresponding medium, followed by anaerobic culture at $30^{\circ} \mathrm{C}$ and $120 \mathrm{rpm}$ for batch and fed-batch fermentations. Samples were taken regularly to measure the concentrations of LA, AA and PA. Additionally, the wild type and the evolved mutants were subjected to transcriptomic, proteomic and metabolomic analyses to further investigate the tolerance mechanism of P. acidipropionici.

RNA sequencing and statistical analysis

Total RNA of the wild type and the three mutants was extracted using an RNeasy Mini Kit (Qiagen, Hilden, Germany). The concentration and purity of the RNA was measured using a NanoDrop instrument. Then, mRNA was isolated and purified using the Poly (A) Purist ${ }^{\text {TM }}$ MAG Kit (Ambion, USA). The cDNA of the transcriptomes was synthesized using the SMARTer ${ }^{\text {TM }}$ PCR cDNA Synthesis Kit (Clontech, USA). The NEBNext ${ }^{\circledR}$ mRNA Library Prep Reagent Set for Illumina ${ }^{\circledR}$ (NEB, UAS) was performed to construct a cDNA library ( $300 \mathrm{bp}$ ), which was subsequently sequenced on the lllumina Xten platform (PE150 mode). The clean data collected after filtering was aligned to the reference transcript sequences using SOAP2. The alignment was conducted via RPKM conversion to obtain the expression level of the transcript.

\section{$\mathrm{RPKM}=\frac{\text { total exon reads }}{\text { mapped reads (millions) } * \text { exon length }(\mathrm{KB})}$}

DEGs were analyzed using the $R$ package edgeR, and the screening threshold was $P$ value $<0.05, \log F C$ (fold change (condition $2 /$ condition 1 ) for a gene) $\geq 1$ or log FC $\leq-1$. The MA plot and volcano plot for each set of samples were charted to visually display the distribution of the differentially expressed genes. Black dots indicate genes without differential expression, while the red dots are the differentially expressed genes.

Functional enrichment analysis of the differentially expressed genes GO (Gene Ontology) analysis of the DEGs was performed using a hyper-geometric distribution. Each GO term with FDR $\leq 0.05$ was chosen as a significantly enriched GO entry. Significant enrichment by pathway can determine the most important biochemical metabolic pathways and signal transduction pathways related to the DEGs. KEGG (Kyoto Encyclopedia of Genes and Genomes) classification was analyzed using $\mathrm{R}$ software by setting the parameter-fdr to $\mathrm{BH}$ to find pathways (FDR $\leq 0.05$ ) where differential genes are significantly enriched relative to all annotated genes.

$$
p=1-\sum_{i=0}^{m-1} \frac{\left(\begin{array}{c}
M \\
i
\end{array}\right)\left(\begin{array}{c}
N-M \\
n-i
\end{array}\right)}{\left(\begin{array}{l}
N \\
n
\end{array}\right)}
$$

where $\mathrm{N}$ is the number of genes with pathway annotation; $\mathrm{n}$ is the number of differentially expressed genes in $\mathrm{N}$; $\mathrm{M}$ is the number of genes annotated as belonging to a particular pathway; $\mathrm{m}$ is the number of 
differentially expressed genes in M.

Protein extraction and LC-MS/MS Analysis

A high strength ultrasonic processor (Scientz, China) was used to treat the samples on ice three times in the lysis buffer ( $8 \mathrm{M}$ urea, $1 \%$ protease inhibitor mixture). The cell debris was removed by centrifugation at $12,000 \mathrm{~g}$ and $4{ }^{\circ} \mathrm{C}$ for $10 \mathrm{~min}$, and the supernatant was collected. The protein concentration was determined using a BCA kit according to the manufacturer's instructions.

The tryptic peptides resuspended in $0.1 \%$ formic acid (buffer $A$ ) were directly loaded onto a self-made reversed-phase analytical column ( $15 \mathrm{~cm}$ length, $75 \mu \mathrm{m}$ inner diameter). The gradient consisted of $6-23 \%$ buffer B ( $0.1 \%$ formic acid in $98 \%$ acetonitrile) over $26 \mathrm{~min}, 23-35 \%$ in $8 \mathrm{~min}, 35-80 \%$ in $3 \mathrm{~min}$, then hold at $80 \%$ for the last 3 min before reversing to buffer $A$ for re-equilibration. The flow rate was set at constant $400 \mathrm{~nL} \cdot \mathrm{min}^{-1}$ on an EASY-nLC 1000 UPLC system.

The peptides were exposed to a nano-spray ionization (NSI) source followed by tandem mass spectrometry (MS/MS) in a Q Exactive ${ }^{\mathrm{TM}}$ Plus instrument (Thermo Fisher Scientific, USA) coupled online to the UPLC. The applied electrospray voltage was $2 \mathrm{kV}$. The $\mathrm{m} / \mathrm{z}$ scan range was from 350 to 1,800 for full scan, and whole peptides were detected at 70,000 resolution in the OrbiTrap. Peptides were then selected for MS/MS using an NCE setting of 28 , and the fragments were detected at 70,000 resolution in the OrbiTrap. A data-dependent program was performed alternately after an MS scan, followed by 20 MS/MS scans, and eliminated dynamically after $15 \mathrm{~s}$. Automatic gain control (AGC) was set at $5 \mathrm{E} 4$. The first fixed mass was set as $100 \mathrm{~m} / \mathrm{z}$.

Data processing and functional enrichment analysis of the differentially expressed proteins (DEPS) The MaxQuant search engine (v.1.5.2.8) was used to analyze the obtained MS/MS data. Tandem mass spectra were searched against the UniProt Proteome database of P. acidipropionici concatenated with a reverse decoy database. Trypsin/P was designated as the enzyme, allowing up to 2 missed cleavage sites. The mass tolerance of precursor ions was set to $20 \mathrm{ppm}$ in the first retrieval, $5 \mathrm{ppm}$ in the main retrieval and 0.02 Da for fragment ions. Oxidation of methionines was designated as variable modification, while carbamidomethylation of cysteines was designated as a fixed modification. The FDR for modification sites, peptides and proteins was adjusted to $<1 \%$ and the lowest score for peptides was set at $>40$. The DEPs were selected when the difference ratio (mutant I/wild-type, mutant II/wild-type, mutant III/wild-type) was greater than 1.5 (significantly upregulated proteins) or less than 1.15 (significantly downregulated proteins) with a t-test P-value $<0.05$.

The proteins were classified into three categories: biological process, cellular component and molecular function by GO annotation (http://www.geneontology.org/). Two-tailed Fisher's exact test was used to test the enrichment of the DEPs based on all identified proteins from the transcriptome of $P$. acidipropionici. The $p$-values from the test were subjected to negative logarithmic (-log10) conversion. Moreover, KEGG (http://www.genome.jp/kegg/) analysis was performed to predict enriched pathways in which the DEPs were involved using the uniform two-tailed Fisher's exact test. KEGG mapper was used to map the annotation results onto the KEGG pathway database. These pathways were classified into hierarchical categories according to the KEGG website. Items with a corrected $p$-value $<0.05$ were considered significant.

Extraction of intracellular metabolites

Page 12/25 
A pipette was used to accurately transfer $10 \mu \mathrm{L}$ of supernatant into a pre-cooled centrifuge tube and combined with $50 \mu \mathrm{L}$ of the internal standard solution. The supernatant was obtained by adding $200 \mu \mathrm{L}$ of pre-cooled methanol: chloroform (3:1, v/v) mixed solution and centrifuging at $12,000 \mathrm{~g}$ and $4{ }^{\circ} \mathrm{C}$ for 10 min. A sample comprising $200 \mu \mathrm{L}$ of the supernatant was removed to the autosampler vial (Agilent Technologies, Foster City, CA, USA), placed in a vacuum centrifuge concentrator (Labconco, Kansas City, Mo, USA) for 5 min to remove the chloroform solvent, and transferred to a lyophilizator (Labconco, Kansas City, Mo, USA) to freeze completely. At room temperature the sample vial was filled with highpurity nitrogen (Parker Balston, Lancaster, NY, USA) and placed in an automated XploreMET derivatization platform (GERSTEL GmbH and Co., Mulheim, Germany) for silanization. The derivatized sample was automatically injected by the injection arm.

Principal component analysis (PCA)

Feature extraction was performed by analyzing intrinsic relationships of metabolites to achieve dimensionality reduction of the multivariate data. PCA was used to observe extreme singular samples with distinctive metabolic profiles, as well as clustering trends for samples. Each of the principal components was independent of each other. The sample characterized by the first principal component had the most prominent feature and the second principal component was second. Through this dimensionality reduction process, changes in hundreds of metabolites and their metabolic networks could be summarized into several key principal components.

Analytical methods

The optical density at $600 \mathrm{~nm}\left(\mathrm{OD}_{600}\right)$ was measured using a standard spectrophotometer (Ultrospec 3300 pro, Amersham Bioscience) to analyze cell growth. The concentrations of organic acids were determined by high-performance liquid chromatography (HPLC) on an HPX-87H organic acid column (Bio-Rad, USA)

\section{Abbreviations}

PA:propionic acid; LA:Lactic acid; AA:Acetic acid; DEPs:differentially expressed proteins; DEGs:differentially expressed genes; PCA:principal component analysis; FDR:false discovery rate; KEGG:kyoto encyclopedia of genes and genomes; GO:gene ontology; LDH:lactate dehydrogenase; POXB:pyruvate oxidase; MMC:methylmalonyl-CoA carboxyltransferase; PMF:proton motive force; ADI:arginine deiminase; $\mathrm{CK}$ :carbamate kinase.

\section{Supplementary Information}

Supplementary information

Supplementary information The Supporting Information is available free of charge on the website.

Additional file 1:

Table S1. Descriptions and Sources of strains, plasmids and primers 
Fig S1. A Growth profiles of the wildtype and the mutant $₫$ under different $\mathrm{pH}$ a pH 7, b pH 6, c pH 5, d pH 4; B Different glucose concentration a $30 \mathrm{~g} \cdot \mathrm{L}^{-1}, \mathrm{~b} 60 \mathrm{~g} \cdot \mathrm{L}^{-1}, \mathrm{c} 90 \mathrm{~g} \cdot \mathrm{L}^{-1}, \mathrm{~d} 120 \mathrm{~g} \cdot \mathrm{L}^{-1}$; C Different oxygen content a $0 \%$, b $10 \%$, c $21 \%$.

\section{Declarations}

\section{Acknowledgements}

Not applicable.

\section{Authors' contributions}

TTL and QRZ designed all the experiments. TTL conducted engineered P. acidipropionici. YL and LYZ performed physiological and fermentation profiles. $\mathrm{HH}$ and $\mathrm{LJ}$ implemented multi-omics sequencing. TTL and LYZ drafted this manuscript. LJ and QRZ revised this manuscript. All authors read and approved the final manuscript.

\section{Funding}

This work was supported by the National Key R\&D Program of China (2018YFC1602800), the National Science Foundation of China (31922070, U1603112), the Natural Science Foundation of Jiangsu Province (BK20171461, BK20180038), the Jiangsu Synergetic Innovation Center for Advanced BioManufacture (XTE1838), and the National High Technology Research and Development Program of China (2012AA021705).

\section{Availability of data and materials}

All data generated or analyzed in this study are available in this article and its additional file.

\section{Ethics approval and consent to participate}

Not applicable.

\section{Consent for publication}

All authors provide consent for publication of the manuscript in Biotechnology for Biofuels.

\section{Competing interests}

The authors declare that they have no competing interests.

\section{References}

1. Guan N, Zhuge X, Li J, Shin H, Wu J, Shi Z, Liu L. Engineering propionibacteria as versatile cell factories for the production of industrially important chemicals: advances, challenges, and 
prospects. Appl Microbiol Biot. 2015;99:585-600.

2. Yang $\mathrm{H}$, Wang $Z$, Lin $\mathrm{M}$, Yang S. Propionic acid production from soy molasses by Propionibacterium acidipropionici: Fermentation kinetics and economic analysis. Bioresource Technol. 2018;250:1-9.

3. Wang X, Salvachua D, Sanchez I Nogue V, Michener WE, Bratis AD, Dorgan JR, Beckham GT. Propionic acid production from corn stover hydrolysate by Propionibacterium acidipropionici. Biotechnol Biofuels. 2017;10:200.

4. Duarte JC, Valenca GP, Moran PJS, Rodrigues JAR. Microbial production of propionic and succinic acid from sorbitol using Propionibacterium acidipropionici. Amb Express. 2015;5:13.

5. Zhang A, Sun J, Wang Z, Yang S, Zhou H. Effects of carbon dioxide on cell growth and propionic acid production from glycerol and glucose by Propionibacterium acidipropionici. Bioresource Technol. 2015;175:374-381.

6. Jiang L, Cui H, Zhu L, Hu Y, Xu X, Li S, Huang H. Enhanced propionic acid production from whey lactose with immobilized Propionibacterium acidipropionici and the role of trehalose synthesis in acid tolerance. Green Chem. 2015;17:250-259.

7. Wang Z, Jin Y, Yang S. High cell density propionic acid fermentation with an acid tolerant strain of Propionibacterium acidipropionici. Biotechnol Bioeng. 2015;112:502-511.

8. Wallenius J, Pahimanolis N, Zoppe J, Kilpelainen P, Master E, Ilvesniemi H, Seppala J, Eerikainen T, Ojamo H. Continuous propionic acid production with Propionibacterium acidipropionici immobilized in a novel xylan hydrogel matrix. Bioresource Technol. 2015;197:1-6.

9. Zhang A, Yang S. Engineering Propionibacterium acidipropionici for enhanced propionic acid tolerance and fermentation. Biotechnol Bioeng. 2009;104:766-773.

10. Xin Z, Liu L, Shin H, Li J, Du G, Chen J. Improved propionic acid production from glycerol with metabolically engineered Propionibacterium jensenii by integrating fed-batch culture with a $\mathrm{pH}$-shift control strategy. Bioresource Technol. 2014;152:519-525.

11. Liu L, Guan N, Zhu G, Li J, Shin H, Du G, Chen J. Pathway engineering of Propionibacterium jensenii for improved production of propionic acid. Sci Rep. 2016; 6:19963.

12. Aburjaile FF, Madec, MN, Parayre S, Miyoshi A, Azevedo V, Le Loir Y, Falentin H. The long-term survival of Propionibacterium freudenreichii in a context of nutrient shortage. J Appl Microbiol. 2016;120:432-440.

13. Fernandes FAN, Gallao MI, Rodrigues S. Effect of osmotic dehydration and ultrasound pre-treatment on cell structure: Melon dehydration. Lwt-Food Sci Technol. 2008;41:604-610.

14. Purvis JE, Yomano LP, Ingram LO. Enhanced trehalose production improves growth of Escherichia coli under osmotic stress. Appl Environ Microb. 2005;71:3761-3769.

15. Bergholz TM, den Bakker HC, Fortes ED, Boor KJ, Wiedmann M. Salt stress phenotypes in Listeria monocytogenes vary by genetic lineage and temperature. Foodborne Pathog Dis. 2010;7:1537-1549.

16. Dragosits M, Mozhayskiy V, Quinones-Soto S, Park J, Tagkopoulos I. Evolutionary potential, crossstress behavior and the genetic basis of acquired stress resistance in Escherichia coli. Mol Syst Biol. 
2013;9:1.

17. Guan N, Du B, Li J, Shin H, Chen RR, Du G, Chen J, Liu L. Comparative genomics and transcriptomics analysis-guided metabolic engineering of Propionibacterium acidipropionici for improved propionic acid production. Biotechnol Bioeng. 2018;115:483-494.

18. Guan N, Li J, Shin H, Wu J, Du G, Shi Z, Liu L, Chen J. Comparative metabolomics analysis of the key metabolic nodes in propionic acid synthesis in Propionibacterium acidipropionici. Metabolomics. 2015;11:1106-1116.

19. Perez-Clemente RM, Vives V, Zandalinas SI, Lopez-Climent MF, Munoz V, Gomez-Cadenas A. Biotechnological Approaches to Study Plant Responses to Stress. Biomed Res Int. 2013.

20. Guan N, Shin H, Chen RR, Li J, Liu L, Du G, Chen J. Understanding of how Propionibacterium acidipropionici respond to propionic acid stress at the level of proteomics. Sci Rep. 2014;4:6951.

21. Luna-Flores $\mathrm{CH}$, Stowers $\mathrm{CC}$, Cox BM, Nielsen LK, Marcellin E. Linking genotype and phenotype in an economically viable propionic acid biosynthesis process. Biotechnol Biofuels. 2018;11:224

22. Thierry A, Deutsch S, Falentin H, Dalmasso M, Cousin FJ, Jan G. New insights into physiology and metabolism of Propionibacterium freudenreichii. Int J Food Microbiol. 2011;149:19-27.

23. Wang Z, Lin M, Wang L, Ammar EM, Yang S. Metabolic engineering of Propionibacterium freudenreichii subsp. shermanii for enhanced propionic acid fermentation: Effects of overexpressing three biotin-dependent carboxylases. Process Biochem. 2015;50:194-204.

24. Almario MP, Reyes LH, Kao KC. Evolutionary engineering of Saccharomyces cerevisiae for enhanced tolerance to hydrolysates of lignocellulosic biomass. Biotechnol Bioeng. 2013;110:2616-2623.

25. Chang C, Guo ZG, He B, Yao B. Metabolic alterations in the sera of Chinese patients with mild persistent asthma: a GC-MS-based metabolomics analysis Acta. Pharmacol Sin. 2015;36:1356.

26. Villas-Boas SG, Bruheim P. Cold glycerol-saline: The promising quenching solution for accurate intracellular metabolite analysis of microbial cells. Anal Biochem. 2007;370:87-97.

27. Baker-Austin C, Dopson M. Life in acid: pH homelostasis in acidophiles. Trends Microbiol. 2007;15:165-171.

28. Lund P, Tramonti A, De Biase D. Coping with low pH: molecular strategies in neutralophilic bacteria. Fems Microbiol Rev. 2014;38:1091-1125.

29. Srinivasan V, Pierik AJ, Lill R. Crystal structures of nucleotide-free and glutathione-bound mitochondrial ABC transporter atm1. Science. 2014;343:1137-1140.

30. Jeong KC, Hung KF, Baumler DJ, Byrd JJ, Kaspar CW. Acid stress damage of DNA is prevented by Dps binding in Escherichia coli 0157: H7. BMC Microbiol. 2008;8:181.

31. Jin J, Liu S, Zhao L, Ge K, Mao X, Ren F. Changes in ffh, uvrA, groES and dnaK mRNA abundance as a function of acid-adaptation and growth phase in Bifidobacterium longum BBMN68 isolated from healthy centenarians. Curr Microbiol. 2011;62:612-617.

32. Senouci-Rezkallah K, Schmitt P, Jobin MP. Amino acids improve acid tolerance and internal pH maintenance in Bacillus cereus ATCC14579 strain. Food Microbiol. 2011;28:364-372. 
33. Guan N, Li J, Shin H, Du G, Chen J, Liu L. Metabolic engineering of acid resistance elements to improve acid resistance and propionic acid production of Propionibacterium jensenii. Biotechnol Bioeng. 2016;113:1294-1304.

34. Guan N, Liu L, Shin H, Chen RR, Zhang J, Li J, Du G, Shi Z, Chen J. Systems-level understanding of how Propionibacterium acidipropionici respond to propionic acid stress at the microenvironment levels: Mechanism and application. J Biotechnol. 2013;167:56-63.

35. Damiano MA, Bastianelli D, Al Dahouk S, Koehler S, Cloeckaert A, De Biase D, Occhialini A. Glutamate decarboxylase-dependent acid resistance in Brucella spp.: Distribution and contribution to fitness under extremely acidic conditions. Appl Environ Microb. 2015;81:578-586.

36. Cayley S, Record MT. Roles of cytoplasmic osmolytes, water, and crowding in the response of Escherichia coli to osmotic stress: Biophysical basis of osmoprotection by glycine betaine. Biochemistry. 2003;42:12596-12609.

37. Gunasekera TS, Csonka LN, Paliy O. Genome-wide transcriptional responses of Escherichia coli k-12 to continuous osmotic and heat stresses. J Bacteriol. 2008;190:3712-3720.

38. White-Ziegler CA, Um S, Prez NM, Berns AL, Malhowski AJ, Young S. Low temperature (23 degrees C) increases expression of biofilm-, cold-shock- and RpoS-dependent genes in Escherichia coli K-12. Microbiol-Sgm. 2008;154:148-166.

39. Ji Z, Zhang H, Liu H, Yaghi OM, Yang P. Cytoprotective metal-organic frameworks for anaerobic bacteria. P Natl Acad Sci USA. 2018;115:10582-10587.

40. Skory CD, Hector RE, Gorsich SW, Rich JO. Analysis of a functional lactate permease in the fungus Rhizopus. Enzyme Microb Tech. 2010;46:43-50.

41. Ammar EM, Jin Y, Wang Z, Yang S. Metabolic engineering of Propionibacterium freudenreichii: effect of expressing phosphoenolpyruvate carboxylase on propionic acid production. Appl Microbiol Biot. 2014;98:7761-7772.

42. Siero JCW, Strother MK, Faraco CC, Hoogduin H, Hendrikse J, Donahue MJ. In vivo quantification of hyperoxic arterial blood water T-1. Nmr Biomed. 2015;28:1518-1525.

43. Wu Q, Liu T, Zhu L, Huang H, Jiang L. Insights from the complete genome sequence of Clostridium tyrobutyricum provide a platform for biotechnological and industrial applications. J Ind Microbiol Biotechnol. 2017;44:1245-1260.

\section{Figures}




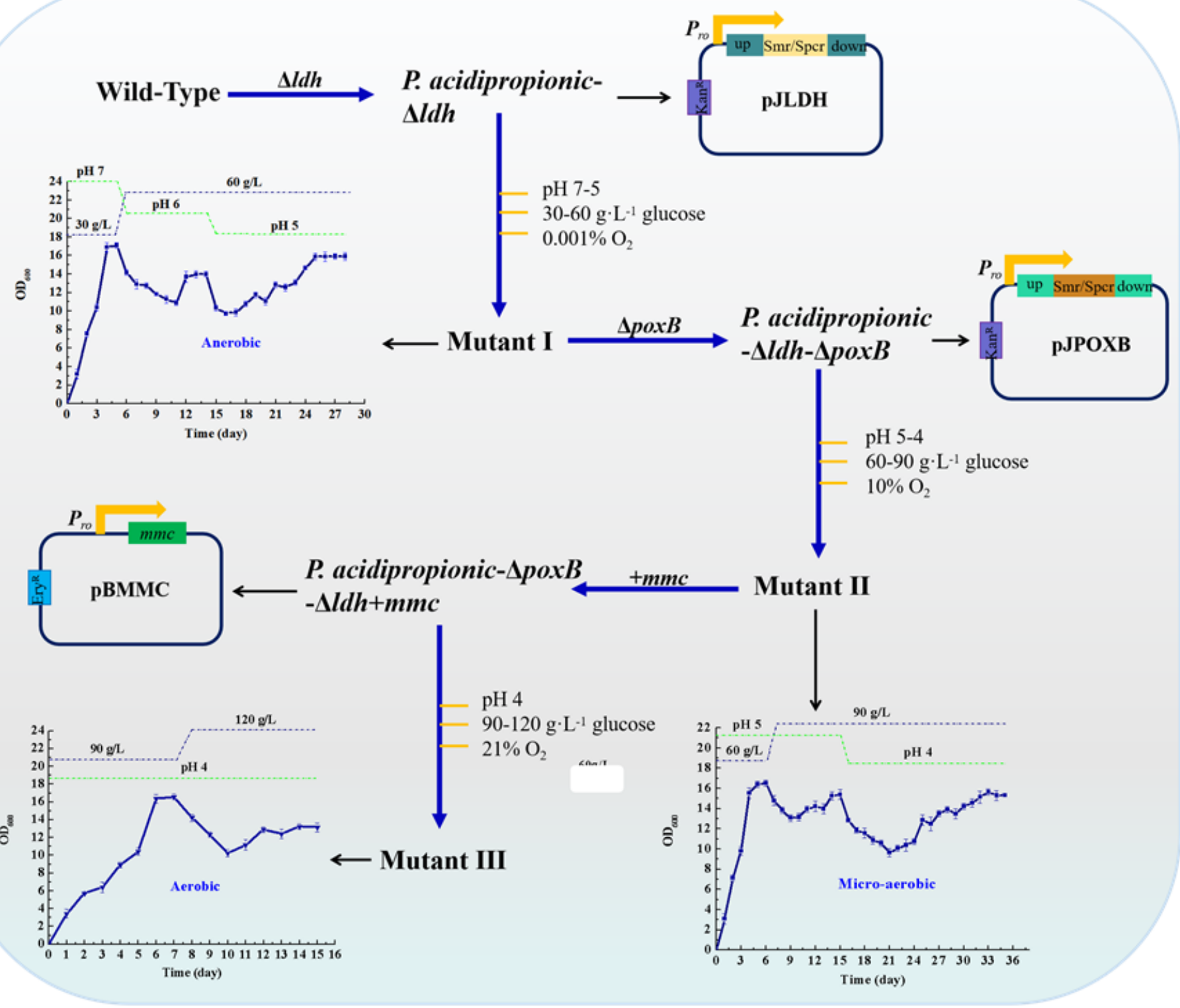

Figure 1

Metabolic and evolutionary engineering of Propionibacterium acidipropionici. 

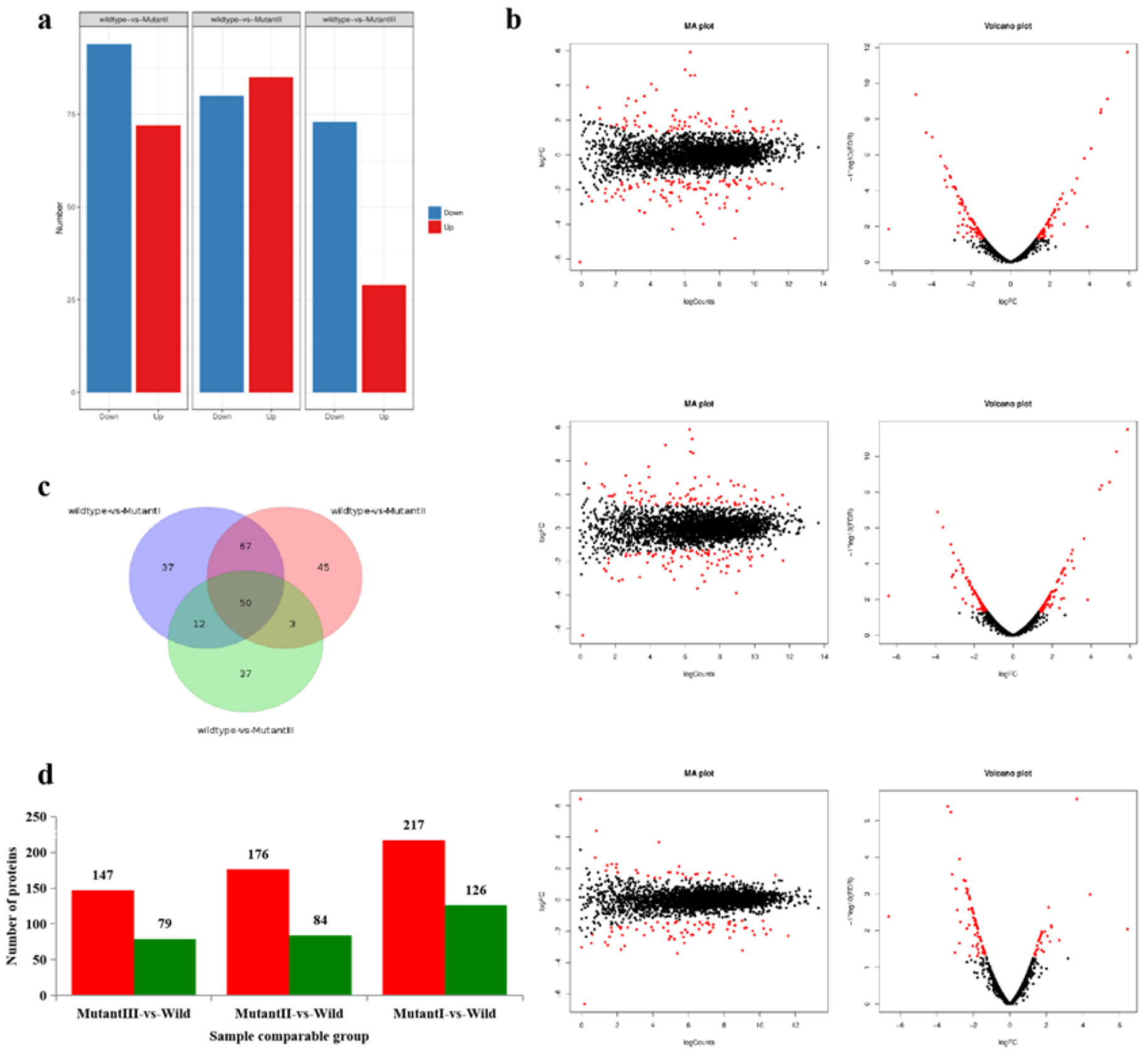

n Up-regulated a Down-regulated

\section{Figure 2}

Differentially expressed genes and proteins a Upregulation and downregulation of differentially expressed genes; $b$ Gene expression MA plot and volcano plot; $c$ Venn diagram of differential genes; $d$ Upregulation and downregulation of differentially expressed proteins. 
a

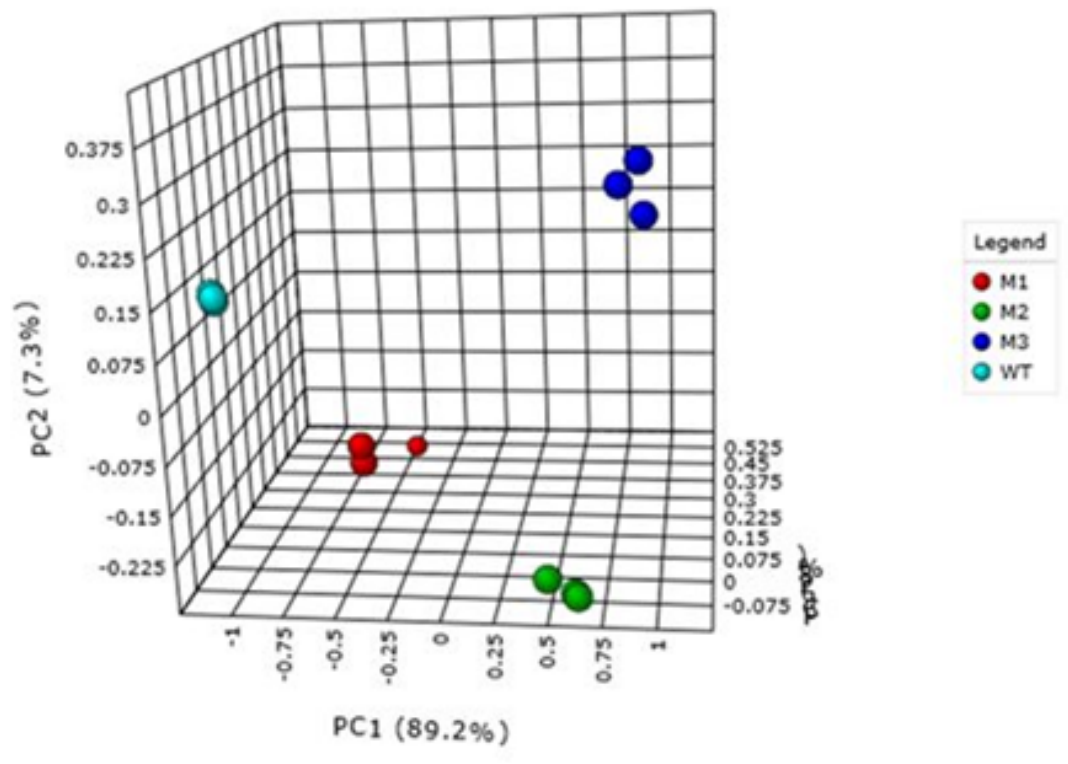

b

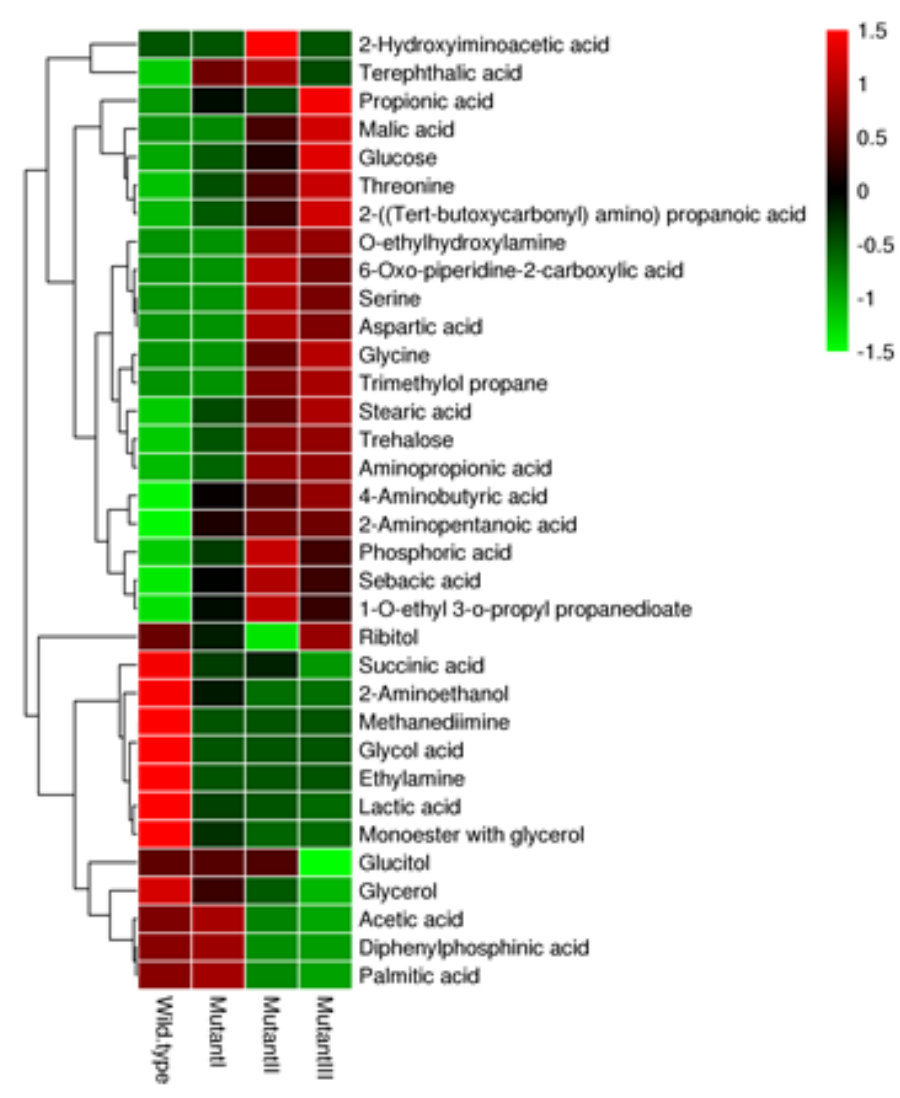

Figure 3

Metabolomic GC-MS analysis of the wild type and the mutants I, II, and III a PCA model based on the metabolites; $b$ Heat map representation of the metabolites. 


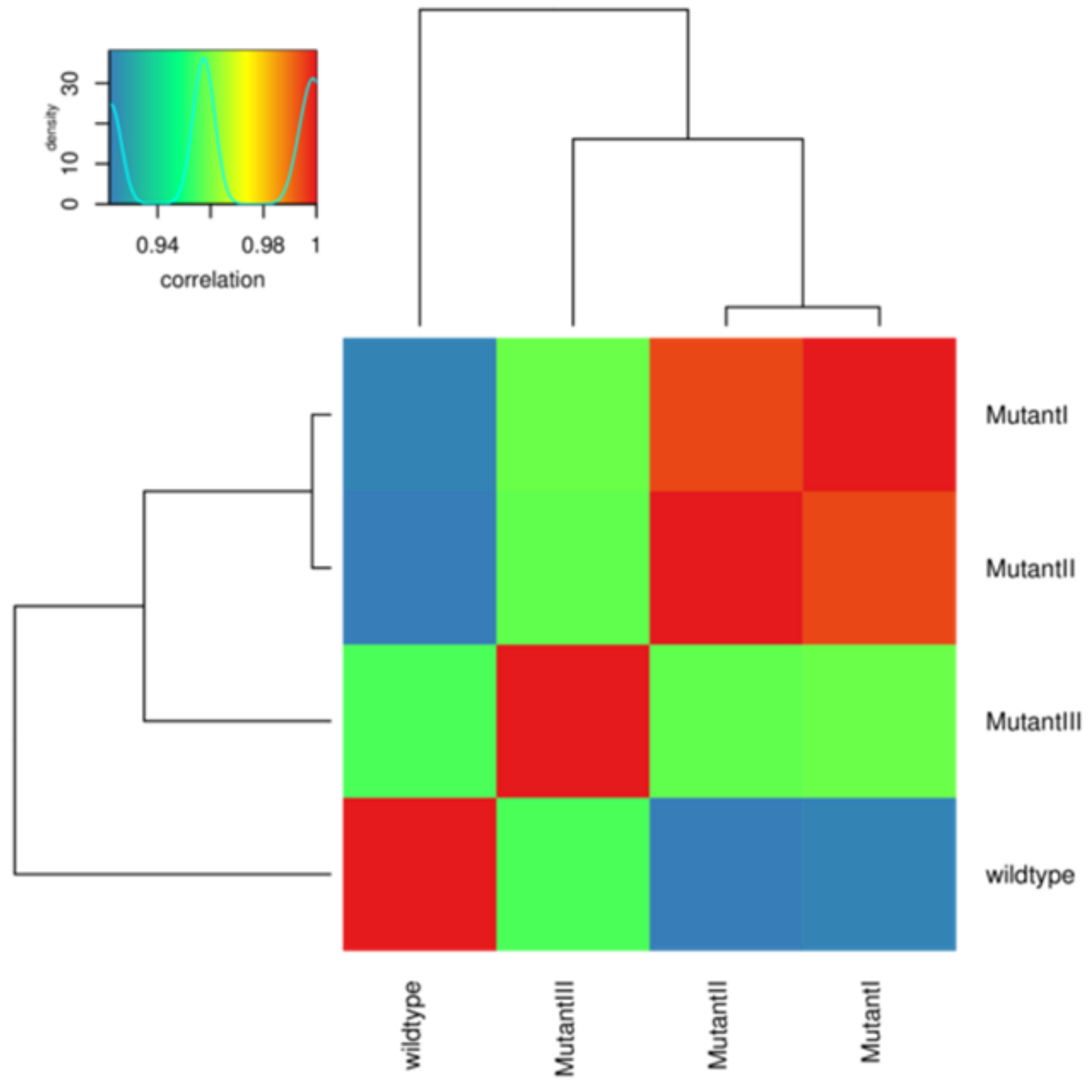

Figure 4

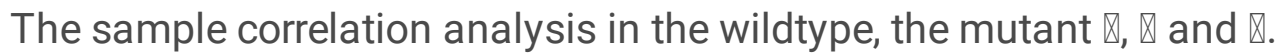


a

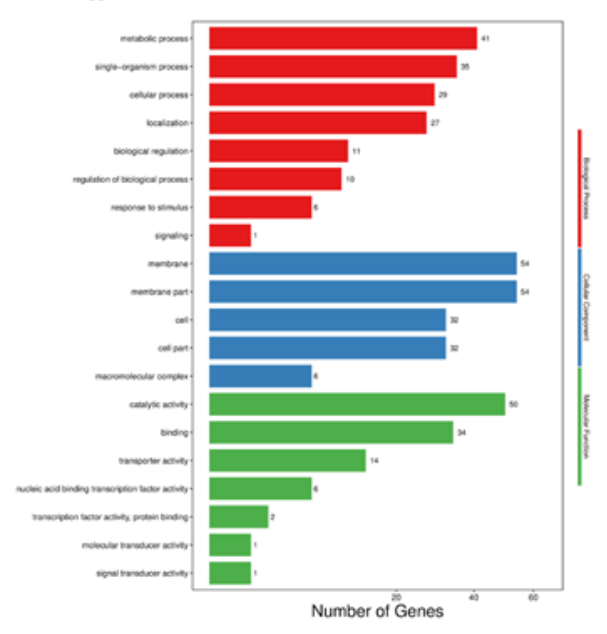

b

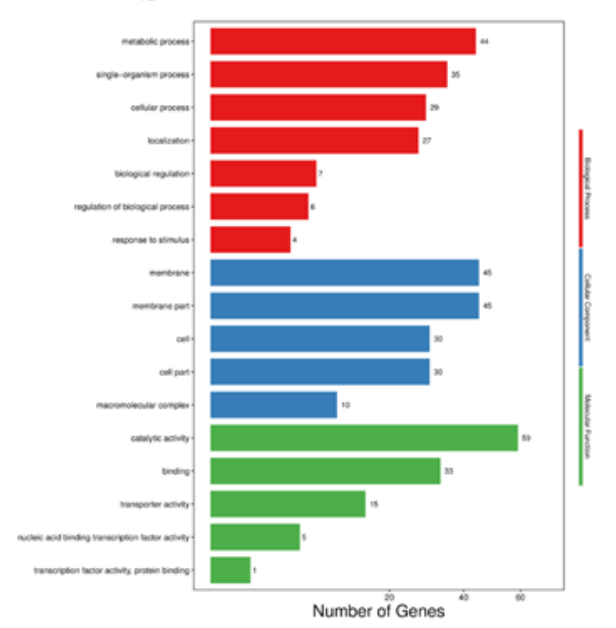

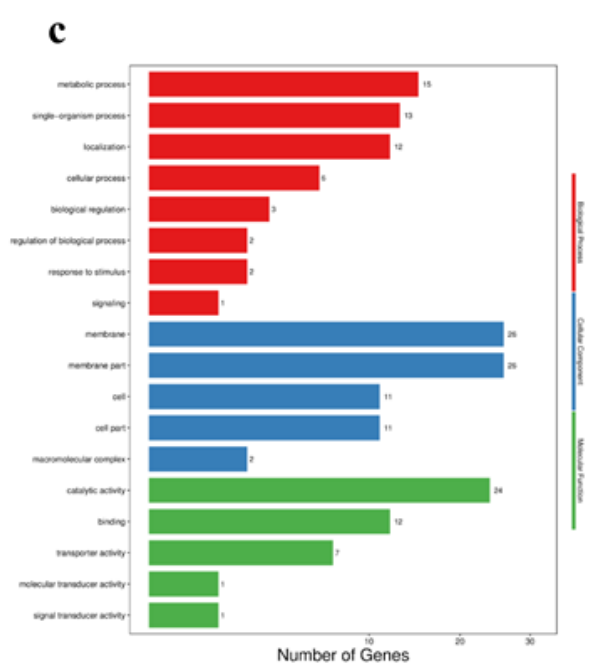

Figure 5

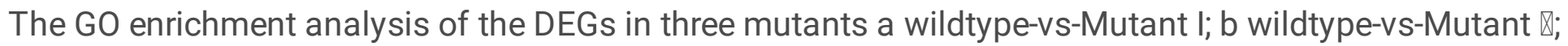

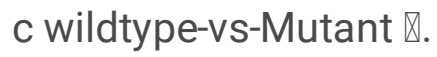
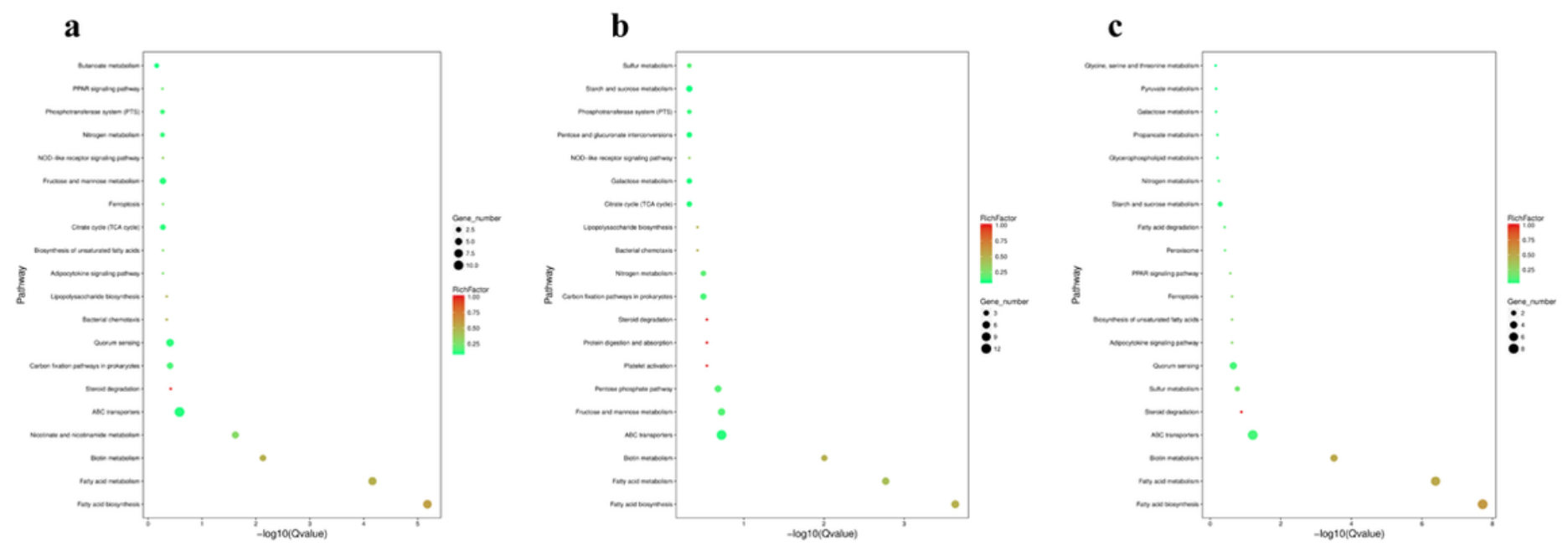

\section{Figure 6}

The KEGG enrichment analysis of the DEGs in three mutants a wildtype-vs-Mutant I; b wildtype-vs-Mutant

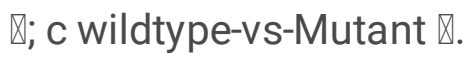


a

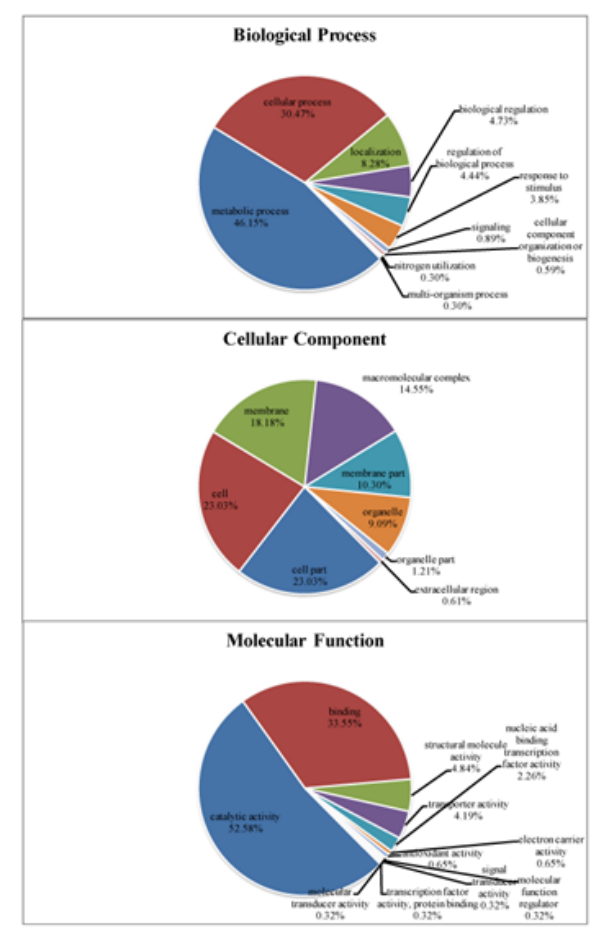

b

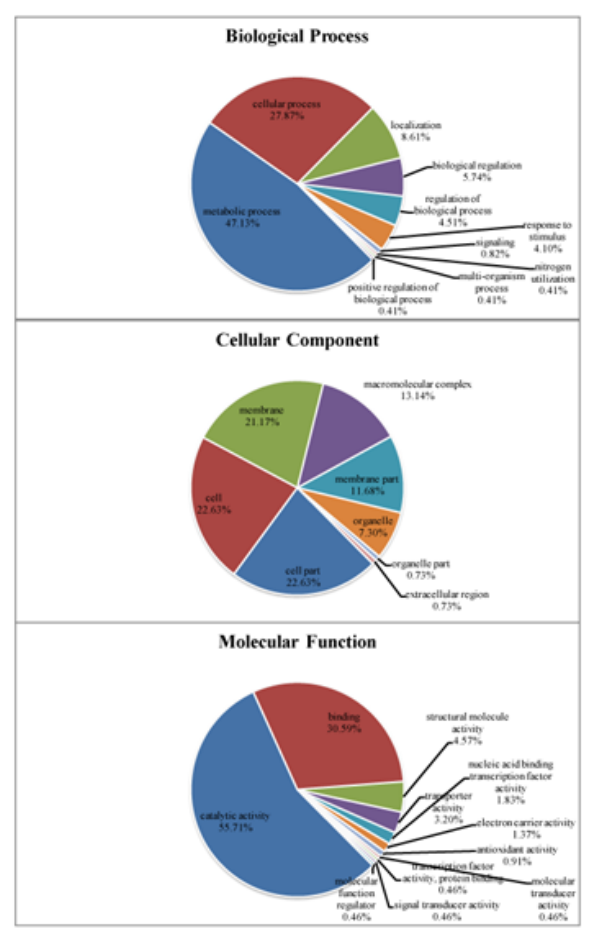

c

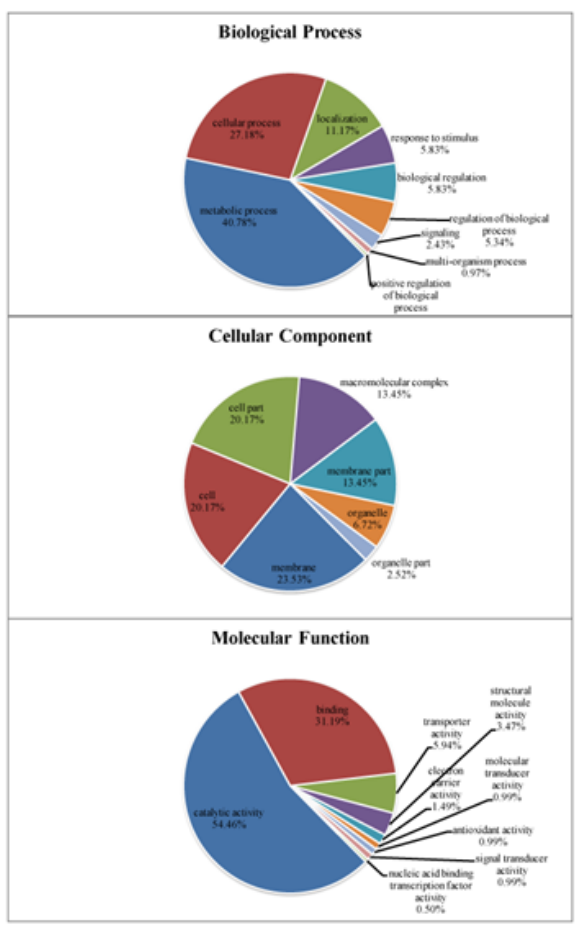

\section{Figure 7}

The GO secondary annotation of the DEPs in three mutants a wildtype-vs-Mutant l; b wildtype-vs-Mutant 邓; c wildtype-vs-Mutant $\nabla$.

A

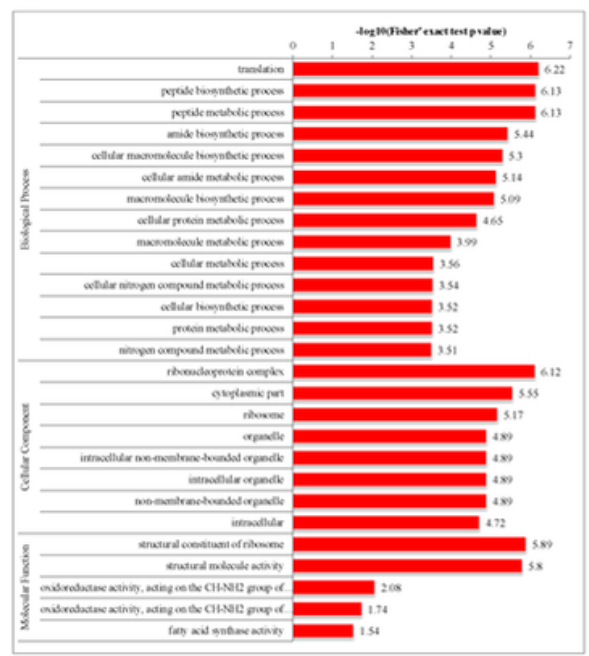

B d

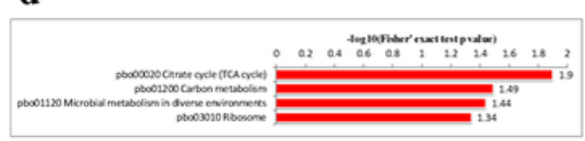

b

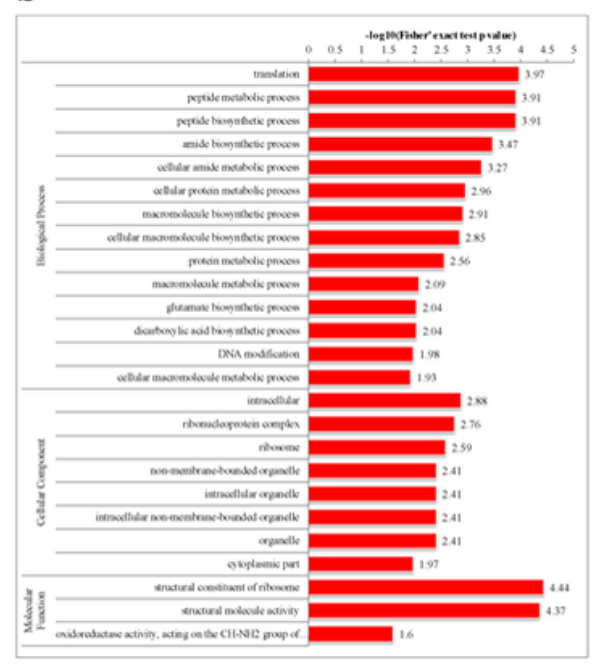

e

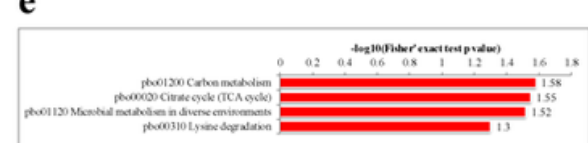

c

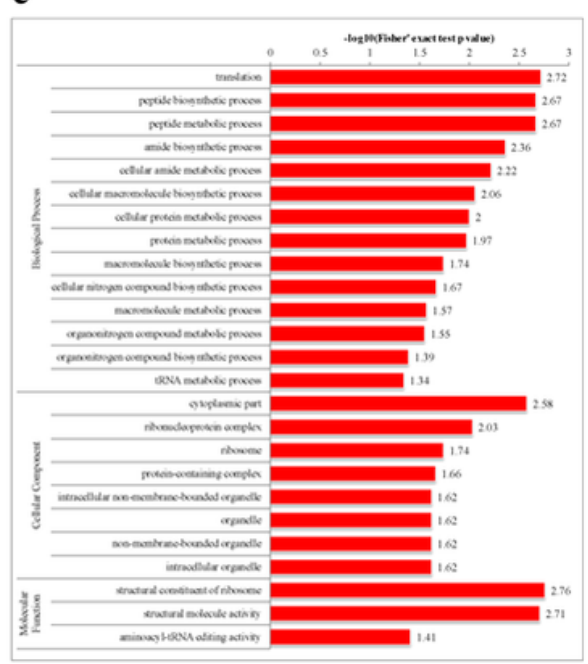

f

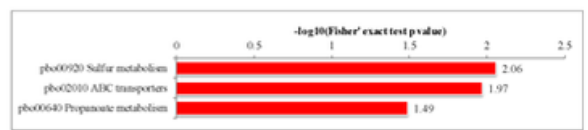

Figure 8 
The GO enrichment A and the KEGG enrichment B of the DEPs in three mutants a and d wildtype-vs-

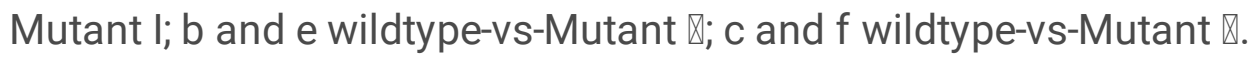

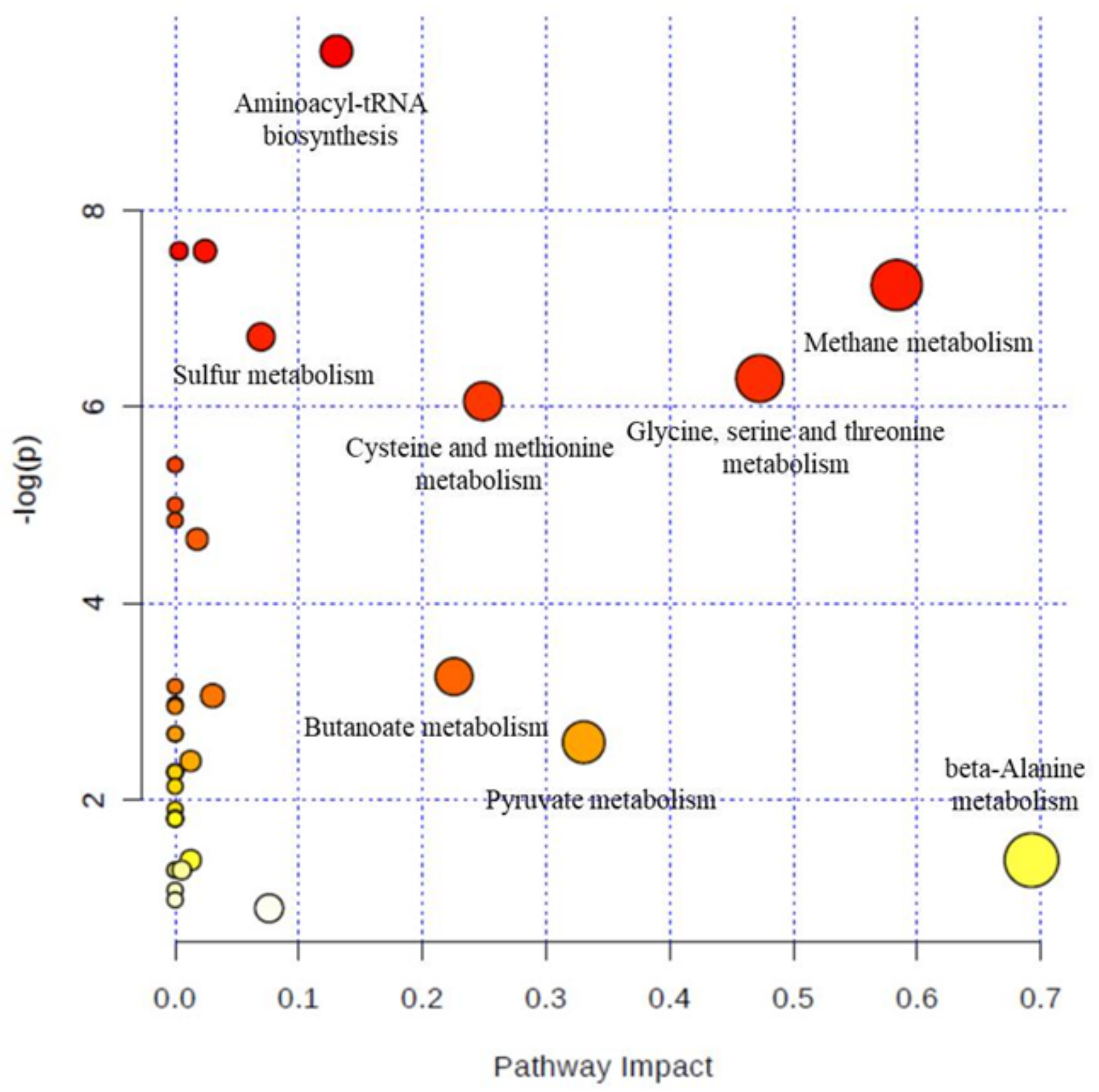

Figure 9

Pathway analysis of the metabolites of the wild type and the mutants I, II and I

\section{Supplementary Files}


This is a list of supplementary files associated with this preprint. Click to download.

- SupplementaryMaterial.doc 